\title{
EVALUATION OF RENAL FUNCTION AFTER PERCUTANEOUS NEPHROSTOMY IN OBSTRUCTIVE UROPATHY DUE TO URETEROPELVIC JUNCTION OBSTRUCTION
}

\author{
MD. FAISAL ISLAM ${ }^{1}$, SM MAHBUB ALAM², MD. BABRUL ALAM ${ }^{3}$, M.A AWAL $^{4}$, MD. AMANUR RASUL ${ }^{2}$
}

${ }^{1}$ Department of Urology, National Institute of Kidney Diseases \& Urology, Dhaka, ${ }^{2}$ Department of Urology, Dhaka Medical College Hospital, Dhaka, ${ }^{3}$ Department of Nephrology, National Institute of Kidney Diseases \& Urology, Dhaka, ${ }^{4}$ Department of Urology, Sher-e-Bangla Medical College, Barisal

\begin{abstract}
:
Objective: The present prospective study was conducted to evaluate the renal function after percutaneous nephrostomy $(P C N)$ in obstructive uropathy due to ureteropelvic junction obstruction. Patients of UPJ obstruction irrespective of sex upto 15 years of age and split renal function (SRF) of the affected kidney $<10 \%$ were enrolled in the study.

Methods: The present prospective study was carried out at the Department of Urology, Dhaka Medical College Hospital from January 2007 to December 2008. The patients having ureteropelvic junction (UPJ) obstruction with poor renal function were the study population. Patients of either sex up to 15 years of age and split renal function $<10 \%$ (unilateral and / bilateral) were enrolled in the study. Patients with other anatomical abnormality of urinary system, concomitant pathologies like stone, pyonephrosis or previous surgical intervention of kidney and/or ureter were excluded from the study. Baseline variables were flank mass, flank pain, side of hydronephrosis, split renal function (SRF), glomerular filtration rate (GFR), specific gravity of urine at the time of PCN. Postoperative follow up variables (at 2, 4 and 6 weeks) were split renal function (SRF), glomerular filtration rate (GFR), specific gravity urine draining through nephrostomy tube, volume of urine through nephrostomy tube and complications. Data were collected using a structured questionnaire (research instrument) containing all the variables of interest. Data were processed and analysed using SPSS (Statistical Package for Social Sciences). The test statistics used to analyse the data were descriptive statistics and Repeated measure ANOVA. For all analytical tests, the level of significance was set at 0.05 and $p<0.05$ was considered significant.
\end{abstract}

Result: The mean age of the patients was $8.6 \pm 3.9$ years and the lowest and highest ages were 3 months and 15 years respectively. The poorly functioning kidneys demonstrated a steady increase in SRF from 3.6\% at baseline to 23\% after 6 weeks PCN $(p<0.001)$. SRF of all children except 4 improved significantly during the period. The mean GFR of poorly functioning kidneys was $3.5 \mathrm{ml} / \mathrm{min} / 1.73 \mathrm{sq}$-meter at baseline which increased to $28.4 \mathrm{ml} / \mathrm{min} / 1.73 \mathrm{sq}$-meter at the end of week 6 . The GFR of 34 children improved (> 10 $\mathrm{ml} / \mathrm{min} / 1.73 \mathrm{sq}$-meter at the 6 week of treatment. Four cases (10.5\%) did not improve. The specific gravity of urine significantly increased from 1.009 at baseline to 1.019 after 6 weeks. The volume of urine at $2^{\text {nd }}$ week was $338 \mathrm{ml} / 24 \mathrm{hour}$ which sharply increased to $363 \mathrm{ml} / 24$ hours at week 4 and nearly $386 \mathrm{ml} / 24$ hours after 6 weeks of PCN $(p<0.001)$.

Conclusion: Present study recommends that as the likelihood of recovery of a severely damaged kidney (SRF < 10\%) with UPJO following PCN is fairly satisfactory; none of the children with poorly functioning kidney should undergo nephrectomy without subjecting them to a PCN trial.

Key word: UPJO, PCN, DTPA renogram

Bangladesh J. Urol. 2014; 17(1): 36-41

Correspondences: Md. Faisal Islam, Department of Urology, National Institute of Kidney Diseases \& Urology, Dhaka, E-mail: faisaluro@gmail.com

Bangladesh J. Urol. 2014; 17(1): 36-41 


\section{Introduction}

Obstructive uropathy is a condition occurring due to blockage of urine flow, resulting in increased pressure within the collecting system leading to kidney injury. Interruption of urine flow results in pain, infection, sepsis and loss of renal function. It is a potentially life threatening condition and immediate measures should be taken to decompress the kidneys. The various modalities available are retrograde stenting, open drainage of kidneys and percutaneous nephrostomy[1]. There are several causes of ureteropelvic junction obstruction. Of them congenital ureteropelvic junction obstruction (UPJO) is the most common cause of hydronephrosis in children. It may be diagnosed by routine prenatal sonograpghy or may present later with symptoms. The management protocol is based on presence of symptoms and, when the conditions are asymptomatic, the function of the affected kidney determines the line of management[2,3]. In patients with unilateral ureteropelvic junction obstruction (UPJO), split renal function (SRF) is measured and in patients with bilateral ureteropelvic junction obstuction, GFR is measured to asses, prognosticate and follow-up. However, no protocol for the management of poorly functioning kidneys (SRF less than 10\%) has been universally accepted[4]. The assessment of potential functional recovery of an obstructed kidney is difficult and inaccurate. Many different techniques such as radiography, pressure flow studies, various functional tests and radionuclide scanning have been used for evaluation of function recoverability following relief of ureteropelvic junction obstruction. None has yielded criteria to predict whether or not reconstructive surgery is advisable for salvage of an obstructed kidney. There are no accurate criteria for potential recovery according to radionuclide evaluation while the kidney is still obstructed[5]. Percutaneous nephrostomy (PCN) has come a long way from the times of William Goodwine. Although it was developed using flouroscopic guidance, ultrasound guided procedure are now safe, easy and effective[4,6,7,8]. Some reports in 1980s described the use of PCN as an easy and safe procedure to determine the recoverability of renal function in obstructed kidneys[5,9]. Renal function may be improved after PCN in obstructed kidneys with SRF $<10 \%$. If SRF becomes more than $10 \%$ after PCN drainage for 4 weeks, pyeloplasty can be done safely instead of nephrectomy[4]. The potential improvement in a kidney with ureteropelvic junction obstruction (UPJO) is likely dependant on multiple factors, such as the severity, duration and time of onset of obstruction in relation to the development of the fetal kidney 3 and the age of the patient at the time of relief of the obstruction $[3,10]$. As there is no alternative means for determining the potential recovery, a trial of percutaneous nephrostomy (PCN) seems logical before deciding whether pyeloplasty or nephrectomy would be needed. The proposed study aimed at determining the proportion of poorly functioning kidney due to UPJO improving following percutaneous nephrostomy seems to be justified.

\section{Materials and Methods}

The present prospective study was carried out at the Department of Urology, Dhaka Medical College Hospital from January 2007 to December 2008. The patients having ureteropelvic junction (UPJ) obstruction with poor renal function were the study population. Patients of either sex up to 15 years of age and split renal function $<10 \%$ (unilateral and / bilateral) were enrolled in the study. Patients with other anatomical abnormality of urinary system, concomitant pathologies like stone, pyonephrosis or previous surgical intervention of kidney and/or ureter were excluded from the study. Baseline variables were flank mass, flank pain, side of hydronephrosis, split renal function (SRF), glomerular filtration rate (GFR), specific gravity of urine at the time of PCN. Postoperative follow up variables (at 2, 4 and 6 weeks) were split renal function (SRF), glomerular filtration rate (GFR), specific gravity urine draining through nephrostomy tube, volume of urine through nephrostomy tube and complications. Data were collected using a structured questionnaire (research instrument) containing all the variables of interest. Data were processed and analysed using SPSS (Statistical Package for Social Sciences). The test statistics used to analyse the data were descriptive statistics and Repeated measure ANOVA. For all analytical tests, the level of significance was set at 0.05 and $p<0.05$ was considered significant.

All patients were evaluated by detail history, physical examination and relevent investigations. Urinalysis, urine culture and sensitivity, complete blood count (CBC), blood urea nitrogen (BUN), serum creatinine and ultrasonography of KUB region was performed. Preoperative IVU was done in all the patients to evaluate the condition of kidney. DTPA renogram was performed to assess the preoperative split renal function (SRF) and glomerular filtration rate (GFR) in all patients. In suspected cases with ureteral dilatation in USG, voiding cystourethrography (VCUG) was done to rule out the 
vesicoureteral reflux (VUR). Documented urinary tract infections were treated with appropriate antibiotic before the procedure. Technetium-99m diethylenetriamine penta-acetic acid (99mTc DTPA) was used for the diuretic renogram according to the standard institutional protocol. Thirty eight subjects fulfilling the criteria underwent PCN drainage for a period of 6 weeks. The PCN was performed under ultrasound guidance after administering local anesthesia supplemented by intravenous sedation with pethidine $(0.5 \mathrm{mg} / \mathrm{kg})$ and promethazine $(0.25 \mathrm{mg} / \mathrm{kg})$. A preprocedural real-time ultrasound was performed to plan the procedure by locating the lower pole calyx from the posterolateral abdominal wall, choosing the closest approach from the skin while avoiding any viscera. The patient was turned on prone position and the skin surface was prepared with povidone iodine. The transducer was inserted into a sterile glove that contains conducting gel, and was secured in place by taping the open end of the glove around the handle. Sterile gel was then placed upon the puncture site. Under local anesthesia and aseptic conditions, a skin nick was made. The initial renal puncture was made with $18 \mathrm{G}$ needle under continuous real time guidance with the free-hand technique. After successful puncture of calyx, using Seldinger technique, a $0.0353 \mathrm{~J}$ tip guide wire was introduced via the needle lumen into the renal pelvis and then the needle is removed. After dilatation of the track with three dilators, an $8 \mathrm{Fr}$ Pigtail catheter was introduced and the guide wire was removed. Dilatation and insertion of nephrostomy tube were all carried out under real time ultrasound guidance. Correct catheter position was determined by free urine drainage. A post procedural scan was performed to look for any complications. No contrast study was done at the time of PCN placement. The catheter was secured to the skin by a silk and was connected to a closed drainage system. Urine sample was collected from drainage bag for specific gravity measurement. Patients were discharged after few hours of observation, with the advice to continue to take the low-dose nitrofurantoin prophylaxis as long as the catheter remained in place. Urine cultures were done weekly, and the patients who developed urinary tract infections received a full course of antibiotics according to the results of the sensitivity tests and were then followed up with low-dose antibiotics during the PCN drainage. After establishment of PCN drainage, followup was performed at 2 weeks, 4 weeks and 6 weeks. During follow up serum creatinine, specific gravity and volume of urine collected through nephrostomy tube were determined. Diuretic renogram with SRF and GFR of affected kidney was done.

\section{Result}

Out of 38 patients 7(18.4\%) were below 5 years, 39.5\% between $5-10$ years and $42.1 \%$ between $10-15$ years. The mean age of the patients was $8.6 \pm 3.9$ years and the lowest and highest ages were 3 months and 15 years respectively. Majority (74\%) of the patients was male giving a male to female ratio of roughly 3:1. Clinical presentation demonstrates that flank mass was the predominant complaint (92.1\%) and flank pain was $52.6 \%$. Flank mass and pain both were present in 19 $(50 \%)$ cases. Twenty six (68.4\%) had left-sided hydronephrosis and the rest 12 (31.6\%) had right-sided hydronephrosis. Complications following PCN showed that $21.1 \%$ of the patients developed fever and $5.2 \%$ haematuria, hematuria and fever both were present in 2 (5.2\%) cases. Evaluation of the subjects in terms of specific gravity shows that the mean urine specific gravity significantly increased during the 6 weeks period following percutaneous nephrostomy (PCN)(table I).

Table-I

Gradual change in specific gravity of urine $(n=38)$

\begin{tabular}{lccc}
\hline Time interval & \multicolumn{2}{c}{ Specific gravity of urine } & p-value \\
\cline { 2 - 3 } & Mean & SD & \\
\hline Baseline & 1.009 & 0.004 & \\
2 weeks & 1.016 & 0.005 & $<0.001$ \\
4 weeks & 1.018 & 0.005 & \\
6 weeks & 1.019 & 0.005 & \\
\hline
\end{tabular}

\# Repeated measure ANOVA statistics was employed to analyze the data.

Mean volume of urine at $2^{\text {nd }}$ week following PCN through nephrostomy tube was $338 \mathrm{ml} / 24$ hours which sharply increased to $363 \mathrm{ml} / 24$ hours at week 4 and $386 \mathrm{ml} / 24$ hours after 6 weeks of PCN. The increase in urinary volume following PCN was statistically significant $(p<$ 0.001 ). The mean split renal function (SRF) of the poorly functioning kidneys experienced a gradual increase in SRFs from 3.6\% at baseline to $16.3 \%$ at week 2, 21.5\% at week 4 and to about $23 \%$ at week 6 (Table II). The improvement of poorly functioning kidneys in terms of SRF was statistically significant $(p<0.001)$. 
Table II

Changes in SRF over time following PCN

\begin{tabular}{lcccccc}
\hline & $\mathrm{N}$ & \multicolumn{2}{c}{ SRF (\%) } & \multicolumn{2}{c}{ p-value } \\
& & Baseline & Week 2 & Week 4 & Week 6 & \\
\hline Poorly functioningkidney & 38 & $3.6 \pm 2.9$ & $16.3 \pm 8.6$ & $21.5 \pm 9.8$ & $22.8 \pm 9.7$ & $<.001$ \\
\hline
\end{tabular}

\# Repeated measure ANOVA statistics was employed to analyze the data and ' $p$ ' refers to overall changes from baseline to endpoint of treatment.

Table III

Changes in GFR following percutaneous nephrostomy (PCN)

\begin{tabular}{lccccc}
\hline & \multicolumn{3}{c}{ GFR(ml/min/sq-meter) } & p-value \\
\cline { 2 - 5 } & Baseline & Week 2 & Week 4 & Week 6 & \\
\hline Poorly functioning kidney & $3.5 \pm 2.8$ & $19.4 \pm 14.1$ & $26.8 \pm 14.7$ & $28.4 \pm 14.6$ & $<0.001$ \\
\hline
\end{tabular}

\# Repeated measure ANOVA statistics was employed to analyse the data and ' $p$ ' refers to overall changes from baseline to endpoint of treatment.

The mean GFR of poorly functioning kidneys was 3.5 $\mathrm{ml} / \mathrm{min} / 1.73 \mathrm{sq}$-meter at baseline which increased sharply to 19.4 and $26.8 \mathrm{ml} / \mathrm{min} / 1.73 \mathrm{sq}$-meter at the end of week 2 and 4 respectively. It then increased insidiously in the next 2 weeks reaching $28.4 \mathrm{ml} / \mathrm{min} /$ 1.73 sq-meter at the end of week 6 (Table-III). The improvement of poorly functioning kidneys following PCN was statistically significant $(p<0.001)$.

Improvement of patients based on SRF $>10 \%$ at the end of 6 weeks shows that nearly $90 \%$ improved and the rest $4(10.5 \%)$ did not improve (Table IV).

Table IV

Improvement of patients based on SRF > 10\% $(n=38)$

\begin{tabular}{lcc}
\hline SRF & Frequency & Percentage \\
\hline$\leq 10 \%$ & 04 & 10.5 \\
$>10 \%$ & 34 & 89.5 \\
\hline
\end{tabular}

\section{Discussion}

Most cases of ureteropelvic junction obstruction (UPJO) are congenital. Acquired conditions such as stone disease, postoperative or inflammatory stricture, or urothelial neoplasm may also present clinically as obstruction at the UPJ level[11]. Hydronephrosis due to congenital UPJO is the most common cause of obstructive uropathy in children. It is also a common cause of abdominal mass in children[4.12]. It occurs more in males. UPJO producing unilateral hydronephrosis predominantly involves the left kidney[12,13]. These findings are supported by the present study which demonstrated a male preponderance $(74 \%)$ and predominant left-sided involvement (68.4\%).

The outcome of the present study was evaluated in terms of volume of urine through nephrostomy tube, specific gravity of urine, changes in SRF and GFR following PCN. Of them SRF and GFR were the main outcome measures. Individual comparison was done to compare the outcome. For convenience the results are discussed with the mean of all the cases. Out of the total 38 cases 4 cases did not improve in terms of specific gravity of urine, volume of urine, SRF and GFR. Even then the calculation was done with all the patients. However, if these 4 cases were not included in the mean then better positive outcome could have been shown.

There is debate regarding the duration of $\mathrm{PCN}$ drainage for evaluation of renal function. Pode et al., (1982) reported functional recovery in 2 of 4 adult kidneys after PCN drainage and recommended a minimum of 4 weeks of drainage to assess recoverability[5]. They described PCN as the most accurate predictive test of kidneys with UPJO and poor function. Flower et al., (1975) described drainage of obstructed kidneys by nephrostomy for an average of 16 days[14]. In another study the optimal period of drainage before maximal recovery was described as two months[15]. In the present study PCN drainage was maintained for 6 weeks. 
The present study demonstrated a modest increase in SRF from $3.6 \%$ at baseline to $22.8 \%$ at week 6 . SRF of $90 \%$ children improved significantly during the period. Of them, 28 (73.3\%) children had SRF $\leq 5 \%$ before PCN, 8 (21.1\%) had SRF $\leq 1 \%$ at baseline. Irving et al., (1987) used PCN drainage in pediatric population to assess the recoverability of function in obstructed kidneys. In their series, nearly 45\% UPJO kidneys recovered enough function to avoid nephrectomy after a trial of PCN drainage. Heloury et al., (1986) reported functional improvement in 6 of 11 poorly functioning UPJO kidneys[12]. They assessed the function by diuretic test and found that only dysplastic kidneys did not improve in function and required nephrectomy. Son et al., (1996) reported significant improvement of SRF in 19 (82.60\%) out of 23 poorly functioning kidneys[16]. Gupta et al., (2001) reported $70 \%$ of the poorly functioning kidneys to improve after a 4 weeks trial of PCN in children[4]. Aziz et al., (2002) reported improvement of split renal function in all children with pre-PCN SRF < $10 \%$ after 6 weeks of PCN drainage. ${ }^{17}$ Poorly functioning kidneys that showed improvement after PCN were likely to retain the improvement in SRF after pyeloplasty[4]. Ransley et al., (1990) recommended a trial of PCN drainage in kidneys with SRF less than $20 \%$ on initial renography[2]. Dhillon, (1998) recommended that kidneys with an initial SRF of less than $10 \%$ would not benefit from PCN drainage and hence should be spared from such trials[18]. The results of the present study are not in conformity with this recommendation, because $90 \%$ (34 out of 38) of the poorly functioning UPJO kidneys (SRF $<10 \%$ ) in children exhibited improvement after PCN. Audry et al., (1996) recommended through his study conducted on neonates and infants with poor renal function due to UPJO that kidneys could be preserved if SRF of poorly functioning kidneys is more than 6\%[19]. Koff and Cambell, (1994) showed that even the most severely hydronephrotic functionally impaired kidneys had good potential for improvement. ${ }^{20}$ Bassiouny, (1992) reported remarkable recovery of renal function in 10 neonates with clinically palpable hydronephrotic non-visualised kidneys with a differential renographic clearance of $<10 \%[21]$.

In the present study the mean GFR of poorly functioning kidneys was $3.5 \mathrm{ml} / \mathrm{min} / 1.73 \mathrm{sq}$-meter at baseline which increased to $28.4 \mathrm{ml} / \mathrm{min} / 1.73 \mathrm{sq}$-meter at the end of week 6 . The GFR of 34 children improved $(\geq 10 \mathrm{ml} / \mathrm{min} /$ $1.73 \mathrm{sq}$-meter) at the 6 week of treatment. Four cases $(10.5 \%)$ did not improve. Out of 38 patients, 11 had baseline GFR $<1 \mathrm{ml} / \mathrm{min} / 1.73$ sq-meter. Of them 10 improved 6 weeks after PCN. Only 1 kidney with GFR < $1 \mathrm{ml} / \mathrm{min} / 1.73 \mathrm{sq}-\mathrm{meter}$ did not improve. These findings suggest that poorly functioning kidney with GFR $<1 \mathrm{ml} /$ $\mathrm{min} / 1.73 \mathrm{sq}$-meter has also the potential to improve following PCN. So poorly functioning kidney even with GFR less than $1 \mathrm{ml} / \mathrm{min} / 1.73$ sq-meter should be preserved until it is decided by PCN that the function of the kidney would not improve after release of UPJ obstruction. Audry et al., (1996) held similar opinion and described PCN as part of management in 17 newborn infants with severe ureteropelvic junction obstruction and showed that kidneys having split creatinine clearance rate more than $1 \mathrm{ml} / \mathrm{min} / 1.73 \mathrm{~m}^{2}$ prior to $\mathrm{PCN}$ should be preserved[19].

Khalaf et al., (2004) studied the methods of prediction of recoverability of renal function after the treatment of adult patients with unilateral obstructive uropathy and a normal contralateral kidney. Kidneys with a renographic GFR of less than $10 \mathrm{~mL} / \mathrm{min} / 1.73 \mathrm{~m}^{2}$ were irreversibly damaged. Improvement or stabilization of function can be expected after relief of obstruction of kidneys with a renographic GFR of $10 \mathrm{~mL} / \mathrm{min} / 1.73 \mathrm{~m}^{2}$ or greater. A preoperative GFR value of $10 \mathrm{~mL} / \mathrm{min} / 1.73 \mathrm{~m}^{2}$ was estimated as the cut-off point that can determine the best prediction of stabilization or improvement of renal function after the relief of obstruction. ${ }^{22}$ However, this comment cannot be compared with young children in whom the potential for recovery of poorly functionally kidney may be better.

In this study urine output through the nephrostomy tube became normal within 6 weeks even with poor renal function (SRF < 10\%) which was consistent with the findings of Dubuisson et al., (1983). Specific gravity of urine also increased during the 6 weeks period following percutaneous nephrostomy (PCN). Baseline specific gravity of urine was 1.009 and after 6 weeks it became 1.019. Though both the values are within normal range the concentrating power of kidney seems to be increased following PCN.

In presence of obstruction with poor function of kidney documented by renography, recoverability of useful renal function cannot reliably be predicted $[9,13]$. Factors influencing renal functional return after prolong periods of obstruction are degree and duration of obstruction, patient's age, greater compliance of collecting system and presence of pyelolymphatic backflow[24]. Regeneration capacity of paediatric renal tissue may be a factor of renal functional return after relieve of obstruction. 


\section{Conclusion}

From the present study it may be recommended that as the likelihood of recovery of a severely damaged kidney (SRF < 10\%) with UPJO following PCN is fairly satisfactory; none of the children with poorly functioning kidney should undergo nephrectomy without subjecting them to a PCN trial.

\section{Conflict of Interest : None Declared}

\section{References:}

1. Sood G, Sood A, Jindal A, Verma DK, Dhiman DS 2006, 'Ultrasonoguided percutaneous nephrostomy for obstructive uropathy in benigbn ad malignant disease', Int Braz J Urol, vol. 32, pp. 281-6.

2. Ransley PG, Dhillon HK, Gordon I 1990, 'The postnatal management of hydronephrosis diagnosed by prenatal ultrasound', J Urol, vol. 144, pp. 584-7.

3. Duckett JW Jr. 1993, 'When to operate on neonatal hydronephrosis', Urology, vol. 42, pp. 617-9.

4. Gupta DK, Chandrasekharam VVSS, Srinivas M and Bajpai M 2001, 'Percutaneous nephrostomy in children with ureteropelvic junction obstruction and poor renal function', Urol, vol. 57, pp. 547-50.

5. Pode D, Shapiro A, Gordon R 1982, 'Percutaneous nephrostomy for assessment of functional recovery of obstructed kidneys', Urology, vol. 19, pp. 482-5.

6. Kehinde EO, Newland CJ, Tery TR, Watkin EM, Butt Z 1993, 'Percutaneous nephrostomy',Br J Urol, vol. 71, pp. 664-6.

7. Pedersen JF 1974, 'Percutaneous nephrostomy guided by ultrasound', J Urol, vol. 112, pp. 157-9.

8. Martino P 2000, 'Ultrasound guided percutaneous nephrostomy', Arch Ital Urol Androl, vol. 72, pp. 324-7.

9. Irving HC, Arthur RJ, and Thomas DF 1987, 'Percutaneous nephrostomy in paediatrics', Clin Radiol, vol. 38, pp. 245-8.

10. Winfield AC, Kirchner SG, Brun ME 1984, 'Percutaneous nephrostomy in neonates, infants, and children', Radiology, vol. 151, pp. 617-9.

11. Jacobs JA, Berger BW, Goldman SM 1979, 'Ureteropelvic junction obstruction in adult with previous normal pyelogram: A report of 5 cases', J Urol, vol. 121, pp. 242.

12. Heloury Y, Schmitt P, Allouch G et al. 1986, 'Treatment of neonatal hydronephrosis by malformation of the ureteropelvic junction: interest of percutaneous nephrostomy', Eur Urol, vol. 12, pp. 224-9.

13. Dubuisson RL, Eichelberger RP, and Jones TB 1983, 'A simple modification of real-time sector sonography to monitor percutaneous nephrostomy', Radiology, vol. 146, pp. 232.

14. Flower JJE, Mears EM and Goldin AR 1975, 'Percutaneous Nephrostomy: technique , indicaton and result', ibid, vol. 6, pp. 428.

15. Kerr WS Jr 1956, 'Effects of complete ureteral obstruction in dogs on kidney function', Am J Physiol, vol. 184, pp. 521.

16. Son H, Kim KM, Choi H 1996, 'Percutaneous Nephrostomy in infants and children.Korean', J Urol, vol. 27, no. 2, pp. 163-8.

17. Aziz MA, Hossain AZ, Banus T, Karim MS, Islam $\mathrm{N}$, Sultana $\mathrm{H}$ et al. 2002, 'In hydronephrosis less than $10 \%$ kidney function is not an indication for nephrestomy in children', Eur J Pediatric Surg, vol. 12. pp. 302-7.

18. Dhillon HK 1998, 'Prenatally diagnosed hydronephrosis: The Great Ormond Street experience', Br J Urol, vol. 81, no. (suppl. 2), pp. 39-44.

19. Audry G, Boyer C, Grapin C, Montagni J P, Gibot LB, Zierej B et al 1996, 'The Value of Split Renal Function in Severe Neonatal and Infant Pelviureteric Obstruction Managed by Percutaneous Nephrostomy', Eur J Pediatric Surg, vol. 6, no. 5. pp. 274-6.

20. Koff SA, and Campbell KD 1994, 'The non-operative management of unilateral neonatal hydronephrosis: natural history of poorly functioning kidneys', J Urol, vol. 152, pp. 593-5.

21. Bassiouny IE 1992, 'Salvage Pyeloplasty in nonvisualizing hydronephrotic kidney secondary to ureteropelvic Junction Obstruction', J Urol, vol. 148, pp. 685-7.

22. Khalaf IM, Shokeir AA, El-gyoushi FI, Amr HS and Amin MM 2004, 'Recoverability of renal function after treatment of adult patients with unilateral obstructive uropathy and normal contralateral kidney: A prospective study', Urology, vol. 64, pp. 664-8.

23. O'Reilly $\mathrm{PH} 1982$, 'Role of modern radiological investigations in obstructive uropathy', Br Med J (Clin Res Ed), vol. 284, pp. 1847-51.

24. Shokeir AA, Shoma AM, Abubieh EA, et al 2002, 'Recoverability of renal function after relief of acute complete ureteral obstruction: Clinical prospective study of the role of renal resistive index', Urology, vol. 59, pp.506-510.

\section{Abbreviations:}

PCN : Percutaneous nephrostomy

SRF : Split renal function

UPJO : Ureteropelvic junction obstruction 